\title{
EXISTENCE AND BOUNDARY BEHAVIOR OF SOLUTIONS FOR BOUNDARY BLOW-UP QUASILINEAR ELLIPTIC PROBLEMS WITH GRADIENT TERMS
}

\section{Chunlian LiU}

Abstract. In this paper, by sub-supersolution methods, Karamata regular variation theory and perturbation method, we study the existence, uniqueness and asymptotic behavior of solutions near the boundary to quasilinear elliptic problem

$$
\left\{\begin{array}{l}
\operatorname{div}\left(|\nabla u|^{m-2} \nabla u\right)=b(x) f(u)\left(1+|\nabla u|^{q(m-1)}\right), \quad x \in \Omega, \\
u>0, \quad x \in \Omega, \\
\left.u\right|_{\partial \Omega}=+\infty,
\end{array}\right.
$$

where $\Omega$ is a bounded domain with smooth boundary in $\mathbf{R}^{\mathbf{N}}(N \geqslant 2), 1<m \leqslant 2,0<q \leqslant$ $m /(m-1) . b \in C^{\alpha}(\bar{\Omega})(\alpha \in(0,1))$ is positive in $\Omega$, and may be vanishing on the boundary, and $f \in C^{1}[0,+\infty), f(0)=0$, is increase on $(0,+\infty)$ and normalized regularly varying at infinity with positive index $p$ and $p+(q-1)(m-1)>0$.

Mathematics subject classification (2020): 35J25, 35B50, 35J40.

Keywords and phrases: Blow-up, quasilinear, elliptic equations, boundary behavior.

\section{REFERENCES}

[1] B. Kawohl, On a family of torsional creep problems, J. Reine Angew. Math., 410, (1990), 1-22.

[2] R. E. Showalter and N. J. Walkington, Diffusion of fluid in a fissured medium with microstructure, SIAM J. Math. Anal. 22, (1991), 1702-1722.

[3] M. C. Pélissier and M. L. Reynaud, Etude d'un modèle mathématique découlement de glacier, C. R. Acad. Sci. Paris Sér. I Math., 279, (1974), 531-534.

[4] C. Bandle and E. Giarrosso, Boundary blow-up for semilinear elliptic equations with nonlinear gradient terms, Adv. Differential Equations, 1, (1996), 133-150.

[5] C. Maderna, C. D. Pagani and S. Salsa, Quasilinear elliptic equations with quadratic growth in the gradient, J. Differential Equations, 97, (1992), 54-70.

[6] A. V. Lair and A. W. Shaker, Classical and weak solutions of a singular semilinear elliptic problem, J. Math. Anal. Appl., 211, (1997), 371-385.

[7] A. V. Lair, A necessary and sufficient condition for existence of large solutions to semilinear elliptic equations, J. Math. Anal. Appl., 240, (1999), 205-218.

[8] A. V. Lair and A. W. Wood, Large solutions of semilinear elliptic equations with nonlinear gradient terms, Int. J. Math. Math. Sci., 22, 4 (1999), 869-883.

[9] Z. Zhang, Large solutions of semilinear elliptic equations with a gradient term: existence and boundary behavior, Commun. Pure Appl. Anal., 12, 3 (2012), 1381-1392.

[10] M. Ghergu, C. Niculescu and V. Rădulescu, Explosive solutions of elliptic equations with absorption and non-linear gradient term, Proc. Indian Acad. Sci. Math. Sci., 112, 3 (2002), 441-451.

[11] C. Bandle and M. Marcus, Large solutions of semilinear elliptic equations: Existence, uniqueness and Asymptotic behavior, J. Anal. Math., 58, (1992), 9-24.

[12] M. Ghergu and V. Rădulescu, Nonradial blow-up solutions of sublinear elliptic equations with gradient term, Commun. Pure Appl. Anal., 3, 3 (2004), 465-474. 
[13] F. Toumi, Existence of blowup solutions for nonlinear problems with a gradient term, International J. Math. Math. Sci., 2006, (2006), 1-11.

[14] Z. Zhang, The asymptotic behavior of solutions with boundary blow-up for semilinear elliptic equations with nonlinear gradient trems, Nonlinear Anal., 62, (2005), 1137-1148.

[15] Z. Zhang, Nonlinear elliptic equations with singular boundary conditions, J. Math. Anal. Appl., 216, (1997), 390-397.

[16] Z. Zhang, Existence of large solutions for a semilinear elliptic problem via sub-supersolutions, Electron. J. Differential Equations, 2, (2006), 1-8.

[17] Y. Chen and M. Wang, Large solutions for quasilinear elliptic equation with nonlinear gradient term, Nonlinear Anal. Real World Appl., 12, 1 (2011), 455-463.

[18] Y. Chen, P. Pang and M. Wang, Blow-up rates and uniqueness of large solutions for elliptic equations with nonlinear gradient term and singular or degenerate weights, Manuscripta Math., 141, (2013), 171-193.

[19] Y. Chen, and M. Wang, Boundary blow-up solutions for elliptic equations with gradient terms and singular weights: Existence, asymptotic behaviour and uniqueness, Proc. Roy. Soc. Edinburgh Sect. A, 141, (2011), 717-737.

[20] Z. Zhang, The existence and boundary behavior of large solutions to semilinear elliptic equations with nonlinear gradient terms, Adv. Nonlinear Anal., 3, (2014), 165-185.

[21] Z. Zhang, Exact boundary behavior of large solutions to semilinear elliptic equations with a nonlinear gradient term, Sci. China Math., 3, (2020), 559-574.

[22] J. L. Kazdan and R. J. Kramer, Invariant criteria for existence of solutions to second-order quasilinear elliptic equations, Comm. Pure Appl. Math., 31, 5 (1978), 619-645.

[23] J. Serrin, The solvability of boundary value problems. In Mathematical developments arising from Hilbert problems, (Northern Illinois Univ., 1974), 507-524. Proc. Sympos. Pure Math. 28, Amer. Math. Soc., Providence, RI, 1976.

[24] H. Bueno, G. Ercole, A. Zumpano and W. M. Ferreira, Positive solutions for the p-Laplacian with dependence on the gradient, Nonlinearity, 25, 4 (2012), 1211-1234.

[25] L. Iturriaga, S. Lorca and J. Sánchez, Existence and multiplicity results for the p-Laplacian with a p-gradient term, NoDEA Nonlinear Differential Equations Appl., 15, 6 (2008), 729-743.

[26] J. Li, J. Yin and Y. Ke, Existence of positive solutions for the p-Laplacian with p-gradient term, J. Math. Anal. Appl., 383, 1 (2011), 147-158.

[27] M. Montenegro and M. Montenegro, Existence and nonexistence of solutions for quasilinear elliptic equations, J. Math. Anal. Appl., 245, 2 (2000), 303-316.

[28] D. Ruiz, A priori estimates and existence of positive solutions for strongly nonlinear problems, J. Differential Equations, 199, 1 (2004), 96-114.

[29] A. Porretta and S. Segura de León, Nonlinear elliptic equations having a gradient term with natural growth, J. Math. Pures Appl., 85, 9 (2006), 465-492.

[30] Y. Du and Z. Guo, Blow-up solutions and their applications in quasilinear elliptic equations, J. Anal. Math., 89, (2003), 277-302.

[31] Z. Yang, Existence of entire explosive positive radial solutions for a class of quasilinear elliptic systems, J. Math. Anal. Appl., 288, (2003), 768-783.

[32] R. Osserman, On the inequality $\Delta u \geqslant f(u)$, Pacific J. Math., 7, (1957), 1641-1647.

[33] Q. Lu, Z. Yang and E. H. Twizell, Existence of Entire Explosive Positive Solutions of Quasi-linear Elliptic Equations, Appl. Math. Comput., 148, (2004), 359-372.

[34] Z. Yang, Existence of explosive positive solutions of quasilinear elliptic equations, Appl. Math. Comput., 177, (2006), 581-588.

[35] C. Liu and Z. Yang, Boundary blow-up quasilinear elliptic problems of the Bieberbach type with nonlinear gradient terms, Nonlinear Anal., 69, (2008), 4380-4391.

[36] C. Liu and Z. Yang, Existence of large solutions for a quasilinear elliptic problem via explosive subsupersolutions, Appl. Math. Comput., 199, (2008), 414-424.

[37] G. Djairo, J. P. Gossez, H. R. Quoirin and P. Ubilla, Elliptic equations involving the p-Laplacian and a gradient term having natural growth, Rev. Mat. Iberoam., 35, 1 (2019), 173-194.

[38] R. Seneta, Regular varying functions, in:Lecture Notes in Mathematics, 508, Springer, 1976.

[39] S. I. Resnick, Extreme Values, Regular Variation, and Point Processes, Springer-Verlag, New York, 1987. 
[40] V. Maric, Regular variation and differential equations, in:Lecture Notes in Mathematics 1726, Springer, 2000.

[41] Z. Guo, Some existence and uniqueness of the positive radial solutions for a class of quasilinear elliptic equations, Appl. Anal., 47, 1 (1992), 173-190.

[42] Z. Guo, Some existence and multiplicity results for a class of quasilinear elliptic eigenvalue problems, Nonlinear Anal., 18, (1992), 957-971.

[43] D. Gilbarg and N. S. Trudinger, Elliptic Partial Differential Equations of Second Order, 3nd edition, Springer-Verlag, Berlin, 1998.

[44] Z. Yang, B. Xu and M. Wu, Existence of positive boundary blow-up solutions for quasilinear elliptic equations via sub and supersolutions, Appl. Math. Comput., 188, 1 (2007), 492-498. 